\title{
Correspondence
}

\section{Addressing physician resources for chronic pain management}

To the Editor:

The article by Veillette et al. ${ }^{1}$ in the June/July issue of the Canadian Journal of Anesthesia draws attention to the serious problem of under funding of chronic pain management in Canada. Québec is not unique in its scarcity of multidisciplinary clinics and lengthy waiting lists. Large areas of Ontario have no access to any chronic pain specialists, let alone multidisciplinary clinics, and waiting lists have ballooned to up to two years in other provinces. In this writer's opinion, the situation will continue to deteriorate unless national and provincial strategies are undertaken promptly.

As Veillette and collaborators state, chronic pain is not rare in Canada with an overall prevalence of $29 \%$ and considerably higher in those over 60 . While demand is increasing, the supply of pain specialists is dwindling. Physicians treating chronic pain are retiring/leaving practice and are not being replaced. A survey I conducted electronically in May, 2005 of 13 university-affiliated Pain Clinic Directors across Canada found that there were only 12 to 15 postgraduate trainees in Chronic Pain Management in the country and some were planning to return to their homeland after training. In the past few years, in South-western Ontario alone, three have left pain practice and three others are on the verge of retiring.

To address the impending crisis effectively will require a multi-pronged attack including the following components:

1) Increased education in Chronic Pain Management at the undergraduate and postgraduate level particularly in Family Medicine, Physical Medicine, Rheumatology, Neurology and Psychiatry residencies. Currently, only Anesthesiology requires mandatory training in this area, yet for the reasons outlined in the article, only a small number of certified anesthesiologists are involved in any form of chronic pain service. Family doctors will continue to provide the mainstay of treatment but are not being adequately trained or supported in the task.

2) Improved provincial insurance fee structure to allow physicians to spend the time necessary to deal with the needs of complex chronic pain patients.

3) Envelope funding from provincial Ministries of Health to support the allied health services required in multidisciplinary pain clinics.

We cannot afford multidisciplinary care for all chronic pain patients; fortunately all do not need it. It is also possible that with greater awareness of the relationship between acute and chronic pain, relatively simple low-cost strategies could be implemented to identify and treat patients earlier who are at high risk of developing persistent pain.

Pat Morley-Forster MD FRCPC

University of Western Ontario, London, Canada

E-mail: pat.morley-forster@sjhc.london.on.ca

\section{Reference}

1 Veillette $\Upsilon$, Dion D, Altier N, Choinière M. The treatment of chronic pain in Québec: a study of hospitalbased services offered within anesthesia departments. Can J Anesth 2005; 52: 600-6.

\section{General anesthesia for a patient with alternating hemiplegia of childhood}

To the Editor:

Alternating hemiplegia of childhood (AHC) is a rare neurological disorder, diagnosed clinically, usually after 18 months. It is characterised by attacks of unilateral hemiplegia, with variable duration and severity. Seizures, occulomotor disturbances and cognitive impairment, can occur. It was first described by Verret in 1971 and is a diagnosis of exclusion and of unknown etiology. ${ }^{1}$

A pregnant 37-yr-old woman with AHC presented at 13 weeks gestation for a termination of pregnancy for severe fetal malformation. Alternating hemiplegia of childhood had been diagnosed at age 14, after an initial misdiagnosis of myasthenia gravis. Her attacks regressed during adolescence, being able to control the triggering factors, namely stress, anxiety, exertion and extremes of temperature. Two previous volatile anesthetics were followed by extreme limb weakness, and difficulty swallowing. Symptoms in both cases resolved without intervention, although the cause was unclear. A general anesthetic performed in 2000 for a Cesarean section was performed with propofol as a target controlled infusion (TCI). A modified rapid 
sequence was performed with rocuronium and alfentanil. She recalled no weakness postoperatively.

At the time of the current presentation, she had brisk reflexes, and a positive Babinski sign on the right with no focal weakness. She was otherwise well, and had a normal airway examination.

She was fasted, and premedicated with ranitidine, metoclopramide and sodium citrate.

The anesthetic machine was prepared according to standard malignant hyperthermia protocol to avoid the potential risk of rhabdomyolysis. Anesthesia was induced with midazolam and fentanyl followed by a propofol TCI. A size 4 laryngeal mask airway was inserted and she remained spontaneously breathing only oxygen for the ten-minute procedure. Routine observations including temperature were stable throughout. At the time of discharge three hours later, she walked unaided, easily.

Evidence suggests that AHC is a channelopathy, sometimes with mitochondrial abnormalities. ${ }^{2}$ Flunarizine, a selective calcium channel blocker, appears to have some success in reducing duration and frequency of attacks. ${ }^{3}$ There has only been one case report of AHC and anesthesia, describing this same patient, whilst she was having her Cesarean section. ${ }^{4}$ For this patient, total $i v$ anesthesia appeared the safest option. She was fearful that the stress of a regional anesthetic could trigger an attack. As she was slim and well fasted, using an laryngeal mask airway was deemed appropriate, and muscle relaxants could thus be avoided. ${ }^{5}$ There are only 250 documented cases of alternating hemiplegia in the world, although under-diagnosis is probably common. Due to the rarity of the disease, and its unusual presentation, patient care would likely benefit from establishment of an international database.

Rina Mehrotra FRCA

Greenlane Hospital, Greenlane, Auckland,

New Zealand

E-mail: rina_london@yahoo.com

\section{References}

1 Verret S, Steele JC. Alternating hemiplegia of childhood: a report of eight patients with complicated migraine beginning in infancy. Paediatrics 1971; 47: 675-80.

2 Casaer P, Azou M. Flunarizine in alternating hemiplegia in childhood. Lancet 1984; 11: 579.

3 Mikati MA, Kramer U, Zupanc ML, Shanahan RJ. Alternating hemiplegia of childhood: clinical manifestations and long term outcome. Pediatr Neurol 2000; 23 134-141
4 Parris-Piper TW. Caesarean section under general anaesthetic in a woman with alternating hemiplegia of childhood. Int J Obstet Anesth 2002; 11: 317-20.

5 Han TH, Brimacombe J, Lee EJ, Yang HS. The laryngeal mask airway is effective (and probably safe) in selected healthy parturients for elective cesarean section: a prospective study of 1067 cases. Can J Anesth 2001; 48: 1117-21.

\section{Dry tap and spinal anesthesia}

To the Editor:

A major advantage of spinal anesthesia is its definitive endpoint i.e., the free flow of cerebrospinal fluid (CSF). ${ }^{1}$ Occasionally the needle is felt to be in the correct space, but on withdrawing the stylet there is no CSF flow ('dry tap'). Common sense dictates that the procedure be repeated, but if the outcome remains the same and the patient refuses general anesthesia the options are limited. Consent was obtained from the hospital's Research and Development Committee and the patient was informed of our intention to publish this case history.

A 60 -yr-old obese $(120 \mathrm{~kg})$ male with a fear of general anesthesia, was admitted for removal of an infected pin and plate from his tibia. Past history included a cervical laminectomy four years previously. He had been admitted for the same procedure (removal of pin and plate) a few months earlier. Spinal anesthesia was attempted, but abandoned after seven attempts, with no notation as to the nature of the difficulty experienced. As the patient refused a general anesthetic, surgery was postponed. On the current admission he remained adamant that he would only consent to regional anesthesia. A 24G Sprotte needle (Pajunk, Geisingen, Germany) was introduced into the L3-4 interspace in the sitting position and although a 'give' was felt, no CSF was seen. The procedure was repeated at the L2-3 interspace with the same result. Aspiration using a 2-mL syringe also failed to produce any CSF. At this point we injected $3 \mathrm{~mL}$ of heavy bupivacaine $0.5 \%$ in the L2-3 space and, within ten minutes he had a sensory block up to T12. Surgery proceeded uneventfully.

Causes of dry tap include a blocked needle, needle in the wrong space, spinal surgery and low CSF pressures. It is possible that in patients with 'absent' CSF or very low CSF pressure, the subarachnoid space is obliterated as the arachnoid "collapses" on the pia. This increases the volume of the subdural space and may explain the absence of CSF. In this setting an epidural may be considered, but is not without poten- 